\title{
Predictors of Progress in the Stage of Adoption of Breast Cancer Screening for Korean Women
}

\author{
Sora Choi, Heeyoung So, Myonghwa Park*
}

\begin{abstract}
Background: It has been proven that an individuals health behavior is determined through a series of processes. This study aimed to assess the stages of adoption of breast cancer screening, and to identify the factors relating to progress through these stages. Materials and Methods: There were 202 female participants aged 2059 years who were living in Chungbuk, South Korea. They were informed of the study purpose and agreed to participate. Data were collected from October 2010 to January 2011 by assessing the breast cancer screening stage, health beliefs, socio-demographic factors, and other facilitating factors. The participant current stage of adoption of breast cancer screening was classified using the Precaution Adoption Process Model (PAPM), and the various PAPM stages were compared with each other to identify factors likely to determine progress between stages. The data were analyzed using the $\chi 2$-test, ANOVA, Duncan test, and multiple logistic regression. Results: Approximately half of all participants were not on-schedule for breast self-examination and mammography (unaware, $9.4 \%$ and $11.4 \%$, unengaged, $8.4 \%$ and $5.0 \%$, undecided, $20.3 \%$ and $17.8 \%$, decided not to act, $1.5 \%$ and $1.0 \%$, decided to act, $13.4 \%$ and $15.3 \%$, respectively). The factors likely to determine the progress from one stage to another were age, marital status, exposure to media information about breast cancer, self-efficacy, and perceived severity. Conclusions: These results suggest that it is necessary to develop a tailored message for breast cancer screening behavior.
\end{abstract}

Keywords: Breast cancer - breast self-examination - mammography - stage of adoption

Asian Pac J Cancer Prev, 16 (7), 2637-2643

\section{Introduction}

Breast cancer is the second most common type of cancer in Korea, following thyroid cancer, and accounts for $15.4 \%$ of all female cancers. In 1996, there were 3,801 cases of breast cancer in Korea, compared to 16,398 cases in 2010, a four-fold increase in 15 years (Korean Breast Cancer Society, 2013). Compared to the West where there are more postmenopausal than premenopausal breast cancer patients, patients that are in the age group of 40-50 years in Korea have the highest rate of breast cancer, and premenopausal breast cancer rate has reached approximately 50\% (Korean Breast Cancer Society, 2013). In addition, women in the 20-30 age group had the highest breast cancer incidence rate when examining the cumulative new patient ratio of each age group (National Health Insurance Corporation, 2009). The incidence of breast cancer in young women under 35 years is higher in Korea than in the West, their survival rates are lower, and their recurrence rates are higher than the 40-50 age group (Kim et al., 2007). Breast cancer is therefore an increasingly major health concern, not only for women in the 40-50 age group, but also for those aged 20-30 years.

In order to have a good prognosis, it is very important to discover and treat breast cancer at an early stage, where the survival rate is approximately $98.4 \%$ (Korean Breast Cancer Society, 2013). Indeed, the survival rate decreases with an increase in breast cancer severity. According to the breast cancer screening recommendations of the Korean Breast Cancer Society (2013), after age 30 years, monthly breast self-examination (BSE) is recommended; after age 35 , clinical examination by a doctor every 2 years is recommended; and after age 40, clinical examination and mammography every 1-2 years is recommended. The diagnosis by BSE is low in sensitivity compared to the other examination methods and has not been found to contribute to reduction in mortality.

Nevertheless, BSE is easy, simple, highly accessible, can help detect breast cancer in the period between regular check-ups, and increases interest in the screening of breast cancer given, because young women tend to avoid clinical examination by physicians. In addition clinical examinations are also low in sensitivity when used as a single method. However, when combined with mammography, clinical examinations are known to improve the breast cancer detection rate slightly although much research remains to be done (Suh and Kim, 2010). Mammography is the only method that has been proven effective for the early detection of breast cancer in largescale clinical trials (Nattinger, 2010). Therefore, BSE 
and mammography are the most appropriate methods for early detection of breast cancer in asymptomatic people. Breast cancer screening behaviors such as BSE and mammography are influenced by sociod-emographic factors such as age, marital status, education level, and income level (Ergin et al., 2012; Jerome-D'Emilia, 2014). These behaviors also influenced by beliefs towards sensibility, severity, helpfulness, barriers and self-efficacy factors (Baysal and Polat, 2012; Jerome-D’Emilia, 2014), and facilitating factors such as family history of breast cancer, screening recommendations by an acquaintance or a health care provider, and the utilization of mass media (Han and Chung, 2006; Ergin et al., 2012). Previous studies reported on how subjective beliefs, attitudes, and abilities of individuals impact breast cancer screening behavior. However, there are limitations to determining the psychological readiness of individuals, such as their awareness, attitudes, and intentions to practice breast cancer screening. Thus, a study that uses a stage model of behavioral changes to systematically assess the phases of changes in breast cancer screening behavior, and is further able to develop an appropriate intervention strategy based on the characteristics of each phase, is necessary. The Precaution Adoption Process Model (PAPM) is based on the principle that individuals' health behavior happens through a series of processes, and on a theory of behavioral change that suggests that people make decisions, such as adopting new preventive measures or stopping risky behaviors, deliberately (Weinstein et al., 2008). In particular, this theory focuses on explaining an individual's process of making decisions of health behavior in the psychological sense. As such, it examines whether it is the lack of recognition of the problem, a lack of attention and personal recognition, or a decision to not change the behavior as the reason for ignoring changes in an consider an individual's health behavior. Thus, it is appropriate to apply this theory to behavioral changes associated with health risks and low awareness of health behavior (Blalock et al., 1996). This theory is different from other existing theories in that it suggests that people in different stages have qualitatively different behaviors, and that the closer an individual is to action, the bigger the difference between necessary intervention and information (Weinstein et al., 2008). Therefore, this theory is appropriate to explain health behaviors such as acts of breast cancer screening, the way in which individuals are led to make a decision to take action and the way in which they then transfer those decisions into actions. The PAPM has been mainly used in studies such as those aiming to prevent osteoporosis (Blalock et al., 1996) and radon testing in homes (Weinstein et al., 1998). Recently, several studies have reported on the factors affecting the practical levels of screening cancer using this model (Costanza et al., 2005; Kye et al., 2006), but studies that take into account BSE and the whole process of mammography, such as screening intentions, action and the impacting factors, are lacking. Breast cancer screening is a preventive health behavior, and whether individuals undertake it is influenced by their socio-demographic factors, their health beliefs, and other associated factors (Baysal and Polat, 2012; Ergin et al., 2012; Han and
Chung, 2006; Jerome-D'Emilia, 2014). Following each phase of change in breast cancer screening behavior to investigate these factors will be very useful as a way to improve the screening rates for early detection of breast cancer, because it identifies the obstacles to the adoption of breast cancer screening.

Hence, this study aims to provide a basis for the systematic intervention strategies to enhance breast cancer screening behaviors, by identifying the correlation between the stage of adoption of screening and health beliefs, self-efficacy, and facilitating factors. This study especially apply the PAPM targeting to women in the 2030 years age group which the incidence of breast cancer has been gradually increasing, and the targeting women in 40-50 years age group which shows a high incidence of breast cancer as compared to other age groups.

\section{Materials and Methods}

\section{Study design and participant}

This study used a cross-sectional, descriptive design. A total of 202 women aged 20-59 years with no history of breast disease, no current pregnancy or breast-feeding were recruited from two urban areas in Chungbuk, South Korea, between October 2010 and January 2011. All respondents were recruited from the university, health promotion centers, community education centers, or churches. All participants were informed on the purpose of the study and that participation was voluntary and anonymous. Persons who agreed to take part in the survey were provided with a self-administered, structured questionnaire that took 15 to 20 minutes to complete.

\section{Measures}

PAPM stage of adoption of breast cancer screening: we developed a unique algorithm for assessing PAPM stage of adoption of breast cancer screening relative to the Korean Breast Cancer Society (2013) preferred guidelines, which refer to the prior PAPM stage of adoption scale (Kye, 2009). Each respondent's answer was classified into one of the seven stages: (1) Unaware (had never heard about BSE or mammography), (2) Unengaged (not thinking about getting screened), (3) Undecided (thinking about getting screened), (4) Decided not to act (decided not to perform BSE or mammography in the future), (5) Decided to act(decided to perform BSE or mammography in the future but was not up- to date), (6) Action (had a BSE within the last 6 months or a mammography within the last 2 years), (7) Maintenance (had a BSE regularly once a month within the last 6 months or at least two mammogram within the last 4 years).

Health beliefs: the health beliefs scale, developed by Champion in 1984 and revised in 1993, 1995, 1999, and 2005 was used to determine the BSE and mammography beliefs of women (Champion, 1984; 1993; 1995; 1999; Champion et al., 2005). Validity and reliability for the Korean version of the scale was done by three separate studies (Chong, 2000 unpublished; Lee et al., 2002; Kye, 2009). This self-completed scale consisted of 8 parts and 45 items. These were susceptibility ( 5 items), seriousness ( 7 items), benefits of BSE (4 items), barriers 
of BSE (6 items), self-efficacy of BSE (7 items), benefits of mammography (4 items), barriers of mammography ( 5 items), and self-efficacy of mammography (7 items). Each item had a score ranging from 1 to 5: strongly disagree (1 point), disagree ( 2 points), neutral ( 3 points), agree (4 points), and strongly agree (5 points). Each subscale was evaluated separately, and was not combined in a single total score. In previous studies, this scale showed reliability coefficients ranging between $0.71-0.92$. In the current study, the Cronbach's alpha values ranged between 0.75-0.94.

Socio-demographic and facilitating factors: the survey contained standard questions regarding age, marital or partner status, level of education, and monthly household income. Questions about the respondents' facilitating factors for breast cancer screening behavior included family history of breast cancer, search experience and exposure to mass media information about the hazards of and precautions to take against breast cancer, and whether the recommendation for breast cancer screening had been given by an acquaintance or a health professional.

\section{Statistical analysis}

Frequency analysis and descriptive statistics were applied to the demographic characteristics, health beliefs and the stage of breast cancer screening behavior. Chisquare and one-way ANOVA tests were performed to assess differences in the stages of adoption, while a post-hoc analysis (Duncan test) was used to determine at which stages mean differences existed. A multiple logistic regression analysis was used to assess the factors determining progress between stages. Analyses were performed using SPSS statistical software.

\section{Results}

General characteristics of the study participants (Table 1)

The mean age was 39.4 years (standard deviation, 10.2; range, 20-59; median, 39.5). In our study participants, $69.8 \%$ were married or living with a partner. In addition, the majority of respondents had completed college and $64.4 \%$ had a monthly household income equivalent to more than US $\$ 3,000$. Family history of breast cancer was reported by $32.7 \%$ of respondents. Furthermore, $58.4 \%$ of respondents had received a provider recommendation for breast cancer screening, and $77.7 \%$ had been exposed to mass media information about the hazards of and precautions to take against breast cancer.

\section{PAPM stage of adoption of breast cancer screening} (Table 2)

PAPM stages of BSE and mammography behavioral changes were defined for all 202 respondents, of whom, respectively, $47 \%$ and $49.5 \%$ of participants had performed a BSE within the last 6 months, or had undertaken mammography within the last 2 years. Of the remaining respondents without any prior experience with BSE and mammography, $9.4 \%$ and $11.4 \%$ were in stage 1 (unaware), $8.4 \%$ and $5.0 \%$ were in stage 2 (unengaged), $20.3 \%$ and $17.8 \%$ were in stage 3 (deciding), $1.5 \%$ and $1.0 \%$ of participants were in stage 4 (decided no), and $13.4 \%$ and $15.3 \%$ were in stage 5 (decided yes), respectively.

Classification of socio-demographic factors, facilitating factors and health beliefs by stage of adoption of BSE and mammography (Tables 3 and 4)

There was no association between the level of education, or the monthly household income with the stage of adoption of BSE. There was, however, a nonsignificant association between family history of breast cancer and both BSE and mammography. Respondents in stage 1 (unaware) and stage 3 (undecided) were on average younger than respondents in the other stages of adoption of mammography. Respondents in stage 6 (action) and stage 7 (maintenance) were more likely to be married or living with a partner than those in the other stages of adoption of BSE and mammography. Moreover, those in stages 6 and 7 were also better educated than respondents in the other stages of adoption of mammography. Having

Table 1. Characteristics of Participants and Beliefs on Breast Screening $(\mathbf{n}=\mathbf{2 0 2})$

\begin{tabular}{|c|c|c|}
\hline Characteristics & $\mathrm{n}$ & $\%$ \\
\hline \multicolumn{3}{|l|}{ Age } \\
\hline 20-39 & 101 & 50 \\
\hline $40-59$ & 101 & 50 \\
\hline \multicolumn{3}{|l|}{ Marital status } \\
\hline Not married (or not living with partner) & 61 & 30.2 \\
\hline Married (living with partner) & 141 & 69.8 \\
\hline \multicolumn{3}{|l|}{ Education } \\
\hline$\leq$ High school & 48 & 23.8 \\
\hline$\geq$ College & 154 & 76.2 \\
\hline \multicolumn{3}{|l|}{ Monthly household income } \\
\hline$<\$ 2,000$ & 27 & 13.4 \\
\hline$\$ 2,000-$ to $<\$ 3,000$ & 45 & 22.3 \\
\hline$\geq \$ 3,000$ & 130 & 64.3 \\
\hline \multicolumn{3}{|l|}{ Provider recommendation } \\
\hline No & 84 & 41.6 \\
\hline Yes & 118 & 58.4 \\
\hline \multicolumn{3}{|l|}{ Breast cancer family history } \\
\hline No & 136 & 67.3 \\
\hline Yes & 66 & 32.7 \\
\hline \multicolumn{3}{|l|}{ Exposure to media } \\
\hline No & 45 & 22.3 \\
\hline Yes & 157 & 77.7 \\
\hline \multicolumn{3}{|l|}{ Information search } \\
\hline No & 148 & 73.3 \\
\hline Yes & 54 & 26.7 \\
\hline
\end{tabular}

Table 2. The PAPM Stages of Breast Cancer Screening $(\mathbf{n}=\mathbf{2 0 2})$

\begin{tabular}{lrrrrr}
\hline & \multicolumn{2}{c}{ BSE } & & \multicolumn{2}{c}{ Mammography } \\
\cline { 2 - 3 } \cline { 6 - 7 } \cline { 6 - 7 } & $\mathrm{n}$ & $\%$ & & $\mathrm{n}$ & $\%$ \\
\hline Stage 1: Unaware & 19 & 9.4 & & 23 & 11.4 \\
Stage 2: Unengaged & 17 & 8.4 & & 10 & 5 \\
Stage 3: Undecided & 41 & 20.3 & & 36 & 17.8 \\
Stage 4: Decided not to Act & 3 & 1.5 & & 2 & 1 \\
Stage 5: Decided to Act & 27 & 13.4 & & 31 & 15.3 \\
Stage 6: Action & 82 & 40.6 & & 28 & 13.9 \\
Stage 7: Maintenance & 13 & 6.4 & & 72 & 35.6 \\
\hline
\end{tabular}

*BSE: Breast self-examination; $\mathrm{n}$ : number of respondents 
the recommendation of a provider (acquaintance or health professional) for breast cancer screening was most strongly associated with stage 6 (action) adoption of BSE, and stage 7 (maintenance) adoption of mammography and occurred substantially less among those in stage 1 (unaware) and stage 2 (unengaged) of both BSE and mammography adoption. The proportion of subjects reporting that they had been exposed to mass media related breast cancer awareness was highest in those in stage 6 (action) or stage 7 (maintenance).

The differences in health beliefs according to the stages of adoption of BSE were statistically significant $(\mathrm{p}<.05)$. However, there was no statistical significance in susceptibility, or severity with the different stages of adoption of mammography. The respondents had low perceived susceptibility and severity in stage 2 (unengaged) than in stage 7 (maintenance) adoption of BSE, whereas they had highly perceived self-efficacy in stage 7 (maintenance) than in the pre-action adoption stages of BSE or mammography. Increasing the perceived benefits to BSE was associated with the more advanced stages of adoption.

Table 3. Characteristics of Participants and Beliefs on Breast Cancer Screening Stages for BSE $(\mathbf{n}=202)$

\begin{tabular}{|c|c|c|c|c|c|c|c|c|c|}
\hline Variable & $\begin{array}{c}\text { Stage } 1 \\
\mathrm{n}(\%) \text { or } \\
\mathrm{M} \pm \mathrm{SD}\end{array}$ & $\begin{array}{l}\text { Stage } 2 \\
\mathrm{n}(\%) \text { or } \\
\mathrm{M} \pm \mathrm{SD}\end{array}$ & $\begin{array}{c}\text { Stage } 3 \\
\mathrm{n}(\%) \text { or } \\
\mathrm{M} \pm \mathrm{SD}\end{array}$ & $\begin{array}{c}\text { Stage } 4 \\
\mathrm{n}(\%) \text { or } \\
\mathrm{M} \pm \mathrm{SD}\end{array}$ & $\begin{array}{c}\text { Stage } 5 \\
\mathrm{n}(\%) \text { or } \\
\mathrm{M} \pm \mathrm{SD}\end{array}$ & $\begin{array}{c}\text { Stage } 6 \\
\mathrm{n}(\%) \text { or } \\
\mathrm{M} \pm \mathrm{SD}\end{array}$ & $\begin{array}{c}\text { Stage } 7 \\
\mathrm{n}(\%) \text { or } \\
\mathrm{M} \pm \mathrm{SD}\end{array}$ & $\chi^{2}$ or $\mathrm{F}$ & $\frac{p}{\text { (Duncan test } \dagger \text { ) }}$ \\
\hline \multicolumn{10}{|l|}{ Age } \\
\hline $20-39$ & $12(11.9)$ & $9(8.9)$ & $27(26.7)$ & $0(0.0)$ & $17(16.8)$ & $32(31.7)$ & $4(4.0)$ & \multirow[t]{2}{*}{16.19} & \multirow[t]{2}{*}{0.013} \\
\hline $40-59$ & $7(6.9)$ & $8(7.9)$ & $14(13.9)$ & $3(3.0)$ & $10(9.9)$ & $50(49.5)$ & $9(8.9)$ & & \\
\hline \multicolumn{10}{|l|}{ Marital status } \\
\hline Not married & $9(6.0)$ & $12(8.1)$ & $27(18.1)$ & $3(2.0)$ & 16 (10.7) & $70(47.0)$ & $12(8.1)$ & \multirow[t]{2}{*}{19.39} & \multirow[t]{2}{*}{$0.002 *$} \\
\hline Married & $10(18.9)$ & $5(9.4)$ & $14(26.4)$ & $0(0.0)$ & $11(20.8)$ & $12(22.6)$ & $1(1.9)$ & & \\
\hline \multicolumn{10}{|l|}{ Education } \\
\hline$\leq$ High school & $3(6.2)$ & $4(8.3)$ & $7(14.6)$ & $2(4.2)$ & $6(12.5)$ & $23(47.9)$ & $3(6.2)$ & \multirow[t]{2}{*}{5.22} & \multirow[t]{2}{*}{$0.501 *$} \\
\hline$\geq$ College & $16(10.4)$ & $13(8.4)$ & $34(22.1)$ & $1(0.6)$ & $21(13.6)$ & $59(38.3)$ & $10(6.5)$ & & \\
\hline \multicolumn{10}{|c|}{ Monthly household income } \\
\hline$<\$ 2,000$ & $2(7.4)$ & $2(7.4)$ & 7 (25.9) & $1(3.7)$ & $4(14.8)$ & $9(33.3)$ & $2(7.4)$ & \multirow[t]{3}{*}{6.6} & \multirow[t]{3}{*}{$0.887^{*}$} \\
\hline$\$ 2,000-$ to $<\$ 3$ & $0003(6.7)$ & $2(4.4)$ & $11(24.4)$ & $0(0.0)$ & $8(17.8)$ & $18(40.0)$ & $3(6.7)$ & & \\
\hline$\geq \$ 3,000$ & $14(10.8)$ & $13(10.0)$ & $23(17.7)$ & $2(1.5)$ & $15(11.5)$ & $55(42.3)$ & $8(6.2)$ & & \\
\hline \multicolumn{10}{|c|}{ Provider recommendation } \\
\hline No & $11(13.1)$ & $11(13.1)$ & $22(26.2)$ & $1(1.2)$ & $14(16.7)$ & $22(26.2)$ & $3(3.6)$ & \multirow[t]{2}{*}{18.72} & \multirow[t]{2}{*}{0.005} \\
\hline Yes & $8(6.8)$ & $6(5.1)$ & $19(16.1)$ & $2(1.7)$ & $13(11.0)$ & $60(50.8)$ & $10(8.5)$ & & \\
\hline \multicolumn{10}{|c|}{ Breast cancer family history } \\
\hline No & $14(10.3)$ & $12(8.8)$ & $33(24.3)$ & $2(1.5)$ & $20(14.7)$ & $47(34.6)$ & $8(5.9)$ & \multirow[t]{2}{*}{8.22} & \multirow[t]{2}{*}{$0.206 *$} \\
\hline Yes & $5(7.6)$ & $5(7.6)$ & $8(12.1)$ & $1(1.5)$ & $7(10.6)$ & $35(53.0)$ & $5(7.6)$ & & \\
\hline \multicolumn{10}{|c|}{ Exposure to media } \\
\hline No & $12(26.7)$ & $3(6.7)$ & $12(26.7)$ & $0(0.0)$ & $5(11.1)$ & $11(24.4)$ & $2(4.4)$ & \multirow[t]{2}{*}{20.7} & \multirow[t]{2}{*}{$0.001 *$} \\
\hline Yes & $7(4.5)$ & $14(8.9)$ & $29(18.5)$ & $3(1.9)$ & $22(14.0)$ & $71(45.2)$ & $11(7.0)$ & & \\
\hline \multicolumn{10}{|c|}{ Information search } \\
\hline No & $16(10.8)$ & $15(10.1)$ & $36(24.3)$ & $3(2.0)$ & $19(12.8)$ & $51(34.5)$ & $8(5.4)$ & \multirow[t]{2}{*}{14.12} & \multirow[t]{2}{*}{$0.021 *$} \\
\hline Yes & $3(5.6)$ & $2(3.7)$ & $5(9.3)$ & $0(0.0)$ & $8(14.8)$ & $31(57.4)$ & $5(9.3)$ & & \\
\hline Susceptibility & $1.86 \pm 0.70$ & $1.51 \pm 0.46$ & $1.96 \pm 0.61$ & $1.60 \pm 0.53$ & $1.93 \pm 0.77$ & $1.86 \pm 0.71$ & $2.42 \pm 0.75$ & 2.4 & $\begin{array}{l}0.029 \\
(2<1.5,6<7)\end{array}$ \\
\hline Severity & $2.71 \pm 0.74$ & $2.26 \pm 0.60$ & $3.06 \pm 0.70$ & $2.71 \pm 0.80$ & $2.79 \pm 0.93$ & $2.96 \pm 0.70$ & $3.34 \pm 0.74$ & 3.71 & $\begin{array}{l}0.002 \\
(2<1,5<3,6,7)\end{array}$ \\
\hline Benefit & $3.70 \pm 0.64$ & $3.53 \pm 0.36$ & $3.81 \pm 0.48$ & $3.00 \pm 0.66$ & $4.00 \pm 0.55$ & $3.89 \pm 0.59$ & $4.08 \pm 0.46$ & 3.24 & $\begin{array}{l}0.005 \\
(2<1<35,6,7)\end{array}$ \\
\hline Barriers & $2.26 \pm 0.77$ & $2.25 \pm 0.44$ & $2.22 \pm 0.54$ & $2.22 \pm 0.48$ & $1.75 \pm 0.56$ & $1.81 \pm 0.59$ & $1.83 \pm 0.66$ & 4.44 & $\begin{array}{l}<0.001 \\
(5,6,7<1,2,3)\end{array}$ \\
\hline Self-efficacy & $2.39 \pm 0.62$ & $2.53 \pm 0.55$ & $2.53 \pm 0.64$ & $2.52 \pm 0.81$ & $2.47 \pm 0.55$ & $2.89 \pm 0.65$ & $3.19 \pm 0.65$ & 4.59 & $\begin{array}{l}<0.001 \\
(1,5<2,3<6,7)\end{array}$ \\
\hline
\end{tabular}

*Fisher's exact test; Stage 1 (unaware); Stage 2 (unengaged); Stage 3 (undecided)); Stage 4 (decided not to act); Stage 5 (decided to act); Stage 6 (action); Stage 7 (maintenance); $\dagger$ : Stage 1, 2: Stage 2, 3: Stage 3, 4: Stage 4, 5: Stage 5, 6: Stage 6, 7: Stage 7; BSE: Breast self-examination; n: number of respondents; M: mean; SD: standard deviation 
DOI:http://dx.doi.org/10.7314/APJCP.2015.16.7.2637

Predictors of Progress in the Stage of Adoption of Breast Cancer Screening for Korean Women

Table 4. Characteristics of Participants and Health Beliefs on Breast Cancer Screening Stages for Mammography $(\mathbf{n}=\mathbf{2 0 2})$

\begin{tabular}{|c|c|c|c|c|c|c|c|c|c|}
\hline Variable & $\begin{array}{c}\text { Stage } 1 \\
\mathrm{n}(\%) \text { or } \\
\mathrm{M} \pm \mathrm{SD}\end{array}$ & $\begin{array}{l}\text { Stage } 2 \\
\mathrm{n}(\%) \text { or } \\
\mathrm{M} \pm \mathrm{SD}\end{array}$ & $\begin{array}{l}\text { Stage } 3 \\
\mathrm{n}(\%) \text { or } \\
\mathrm{M} \pm \mathrm{SD}\end{array}$ & $\begin{array}{c}\text { Stage } 4 \\
\mathrm{n}(\%) \text { or } \\
\mathrm{M} \pm \mathrm{SD}\end{array}$ & $\begin{array}{c}\text { Stage } 5 \\
\mathrm{n}(\%) \text { or } \\
\mathrm{M} \pm \mathrm{SD}\end{array}$ & $\begin{array}{c}\text { Stage } 6 \\
\mathrm{n}(\%) \text { or } \\
\mathrm{M} \pm \mathrm{SD}\end{array}$ & $\begin{array}{c}\text { Stage } 7 \\
\mathrm{n}(\%) \text { or } \\
\mathrm{M} \pm \mathrm{SD}\end{array}$ & $\chi^{2}$ or $\mathrm{F}$ & $\frac{p}{\text { (Duncan test } \dagger \text { ) }}$ \\
\hline \multicolumn{10}{|l|}{ Age } \\
\hline $20-39$ & $23(22.8)$ & $8(7.9)$ & $24(23.8)$ & $0(0.0)$ & $21(20.8)$ & $9(8.9)$ & $16(15.8)$ & \multirow[t]{2}{*}{62.3} & \multirow[t]{2}{*}{$<0.001$} \\
\hline $40-59$ & $0(0.0)$ & $2(2.0)$ & $12(11.9)$ & $2(2.0)$ & $10(9.9)$ & $19(18.8)$ & $56(55.4)$ & & \\
\hline \multicolumn{10}{|l|}{ Marital status } \\
\hline Not married & $19(35.8)$ & $4(7.5)$ & $13(24.5)$ & $0(0.0)$ & $9(17.0)$ & $3(5.7)$ & $5(9.4)$ & \multirow[t]{2}{*}{55.66} & \multirow[t]{2}{*}{$<0.001 *$} \\
\hline Married & $4(2.7)$ & $6(4.0)$ & $23(15.4)$ & $2(1.3)$ & $22(14.8)$ & $25(16.8)$ & $67(45.0)$ & & \\
\hline \multicolumn{10}{|l|}{ Education } \\
\hline$\leq$ High school & $5(10.4)$ & $3(6.2)$ & $4(8.3)$ & $2(4.2)$ & $4(8.3)$ & $11(22.9)$ & $19(39.6)$ & \multirow[t]{2}{*}{14.37} & \multirow[t]{2}{*}{$0.018^{*}$} \\
\hline$\geq$ College & $18(11.7)$ & $7(4.5)$ & $32(20.8)$ & $0(0.0)$ & $27(17.5)$ & $17(11.0)$ & $53(34.4)$ & & \\
\hline \multicolumn{10}{|c|}{ Monthly household income } \\
\hline$<\$ 2,000$ & $8(29.6)$ & $2(7.4)$ & $5(18.5)$ & $1(3.7)$ & $2(7.4)$ & $2(7.4)$ & $7(25.9)$ & \multirow[t]{3}{*}{21.84} & \multirow[t]{3}{*}{$0.039^{*}$} \\
\hline$\$ 2,000-$ to $<\$ 3$ & $0004(8.9)$ & $1(2.2)$ & $13(28.9)$ & $0(0.0)$ & $8(17.8)$ & $4(8.9)$ & $15(33.3)$ & & \\
\hline$\geq \$ 3,000$ & $11(8.5)$ & $7(5.4)$ & $18(13.8)$ & $1(0.8)$ & $21(16.2)$ & $22(16.9)$ & $50(38.5)$ & & \\
\hline \multicolumn{10}{|c|}{ Provider recommendation } \\
\hline No & $20(23.8)$ & $6(7.1)$ & $16(19.0)$ & $0(0.0)$ & $16(19.0)$ & $11(13.1)$ & $15(17.9)$ & \multirow[t]{2}{*}{37.31} & \multirow[t]{2}{*}{$<0.001 *$} \\
\hline Yes & $3(2.5)$ & $4(3.4)$ & $20(16.9)$ & $2(1.7)$ & $15(12.7)$ & $17(14.4)$ & $57(48.3)$ & & \\
\hline \multicolumn{10}{|c|}{ Breast cancer family history } \\
\hline No & $17(12.5)$ & $8(5.9)$ & $28(20.6)$ & $2(1.5)$ & $22(16.2)$ & $15(11.0)$ & $44(32.4)$ & \multirow[t]{2}{*}{7.07} & \multirow[t]{2}{*}{$0.299 *$} \\
\hline Yes & $6(9.1)$ & $2(3.0)$ & $8(12.1)$ & $0(0.0)$ & $9(13.6)$ & $13(19.7)$ & $28(42.4)$ & & \\
\hline \multicolumn{10}{|c|}{ Exposure to media } \\
\hline No & $14(31.1)$ & $1(2.2)$ & $7(15.6)$ & $0(0.0)$ & $6(13.3)$ & $6(13.3)$ & $11(24.4)$ & \multirow[t]{2}{*}{19.05} & \multirow[t]{2}{*}{$0.002 *$} \\
\hline Yes & $9(5.7)$ & $9(5.7)$ & $29(18.5)$ & $2(1.3)$ & $25(15.9)$ & $22(14.0)$ & $61(38.9)$ & & \\
\hline \multicolumn{10}{|c|}{ Information search } \\
\hline No & $21(14.2)$ & $8(5.4)$ & $26(17.6)$ & $2(1.4)$ & $19(12.8)$ & $22(14.9)$ & $50(33.8)$ & \multirow[t]{2}{*}{7.69} & \multirow[t]{2}{*}{$0.241 *$} \\
\hline Yes & $2(3.7)$ & $2(3.7)$ & $10(18.5)$ & $0(0.0)$ & $12(22.2)$ & $6(11.1)$ & $22(40.7)$ & & \\
\hline Susceptibility & $2.00 \pm 0.77$ & $1.94 \pm 0.94$ & $1.82 \pm 0.61$ & $1.50 \pm 0.71$ & $1.87 \pm 0.63$ & $1.87 \pm 0.60$ & $1.92 \pm 0.75$ & 0.3 & 0.938 \\
\hline Severity & $2.99 \pm 0.59$ & $2.46 \pm 0.77$ & $2.82 \pm 0.78$ & $2.64 \pm 1.11$ & $2.79 \pm 0.71$ & $3.04 \pm 0.76$ & $2.97 \pm 0.81$ & 1.08 & 0.376 \\
\hline Benefit & $3.97 \pm 0.53$ & $3.88 \pm 0.49$ & $4.01 \pm 0.49$ & $3.50 \pm 0.35$ & $4.19 \pm 0.51$ & $4.11 \pm 0.61$ & $4.26 \pm 0.59$ & 2.12 & 0.053 \\
\hline Barriers & $2.71 \pm 0.75$ & $2.42 \pm 0.66$ & $2.66 \pm 0.68$ & $2.60 \pm 1.70$ & $2.28 \pm 0.72$ & $2.18 \pm 0.95$ & $2.23 \pm 0.74$ & 2.41 & 0.029 \\
\hline Self-efficacy & $3.45 \pm 0.57$ & $3.93 \pm 0.73$ & $3.71 \pm 0.56$ & $3.71 \pm 0.40$ & $3.90 \pm 0.46$ & $3.98 \pm 0.52$ & $4.01 \pm 0.55$ & 3.84 & $\begin{array}{l}0.001 \\
(1<2,35,6<7)\end{array}$ \\
\hline
\end{tabular}

*Fisher's exact test; Stage 1 (unaware); Stage 2 (unengaged); Stage 3 (undecided)); Stage 4 (decided not to act); Stage 5 (decided to act); Stage 6 (action); Stage 7 (maintenance); $\uparrow 1$ : Stage 1, 2: Stage 2, 3: Stage 3, 4: Stage 4, 5: Stage 5, 6: Stage 6, 7: Stage 7; BSE: Breast self-examination; n: number of respondents; M: mean; SD: standard deviation

Table 5. Predictors of Breast Cancer Screening Stages for BSE \& Mammography (n=202)

\begin{tabular}{|c|c|c|c|c|}
\hline \multirow[t]{2}{*}{ Variables } & \multicolumn{2}{|c|}{ BSE } & \multicolumn{2}{|c|}{ Mammography } \\
\hline & $\begin{array}{c}\text { Stage } 3(n=41) \\
\text { versus Stage } 2(n=17) \dagger \\
\text { OR }(95 \% \text { CI })\end{array}$ & $\begin{array}{c}\text { Stage } 6(n=82) \\
\text { versus Stage } 5(n=27) \neq \\
\text { OR }(95 \% \text { CI })\end{array}$ & $\begin{array}{c}\text { Stage } 2(n=10) \\
\text { versus Stage } 1(n=23) \S \\
\text { OR }(95 \% \text { CI })\end{array}$ & $\begin{array}{c}\text { Stage } 6(n=28) \\
\text { versus Stage } 5(n=31) \\
\text { OR }(95 \% \text { CI })\end{array}$ \\
\hline \multicolumn{5}{|l|}{ Age } \\
\hline $\begin{array}{l}20-39 \\
40-59\end{array}$ & & $\begin{array}{c}1 \\
1.98(0.66-5.94)\end{array}$ & & $\begin{array}{c}1 \\
3.60(1.16-11.18)\end{array}$ \\
\hline \multicolumn{5}{|l|}{ Marital status } \\
\hline Married & & $2.21(0.70-6.90)$ & $13.91(1.33-145.48)$ & \\
\hline $\begin{array}{l}\text { Education } \\
\leq \text { High school } \\
\geq \text { College }\end{array}$ & & & & $\begin{array}{c}1 \\
0.31(0.08-1.22)\end{array}$ \\
\hline \multicolumn{5}{|c|}{ Provider recommendation } \\
\hline $\begin{array}{l}\text { No } \\
\text { Yes }\end{array}$ & & $\begin{array}{c}1 \\
2.45(0.91-6.64)\end{array}$ & & \\
\hline \multicolumn{5}{|c|}{ Exposure to media } \\
\hline $\begin{array}{l}\text { No } \\
\text { Yes }\end{array}$ & & & $\frac{1}{25.65(166}$ & \\
\hline Susceptibility & $1.20(0.91-1.58)$ & & & \\
\hline Severity & $1.29(1.06-1.55)$ & & & \\
\hline Benefit & $1.46(0.92-2.31)$ & & & \\
\hline Self-efficacy & & $1.18(1.05-1.34)$ & & \\
\hline
\end{tabular}


of BSE progressed from stage 5 to stage 6 , a higher selfefficacy was significantly associated with stage 6 (OR, 1.18 ; 95\% CI: 1.05-1.34). When we explored the factors that distinguish those who had not been thinking about getting screened (stage 2) from those who had never heard about mammography (stage 1), we again found that the progress in the stage of adoption of mammography from stage 1 to stage 2 was significantly associated with being married or living with partner. Knowledge about mass media information related to the hazards of and precautions against breast cancer was also associated with progression to stage 2 . In addition, we found that being older was significantly associated with progression from stage 5 to stage 6 adoption of mammography.

\section{Discussion}

In this study, we identified predictors of the progress of PAPM stages of adoption for the psychological readiness of individuals such as awareness, attitudes and intentions to practicing breast cancer screening behavior in a primary care population typical of a community.

We assessed the stages of adoption of BSE and mammography in the study population. A little less than half of the respondents reported on-schedule screening for BSE and mammography, while 53\% and $50.5 \%$ did not, respectively (unaware, 9.4\%, 11.4\%; unengaged, 8.4\%, 5.0\%; undecided, $20.3 \%, 17.8 \%$; decided no, $1.5 \%$, $1.0 \%$; decided yes, $13.4 \%, 15.3 \%$, respectively). The rate of breast cancer screening was similar to that reported in other population-based studies (Han and Chung, 2006; Ministry of health and welfare, 2013). However, in this study, women who had never undergone breast cancer screening made up more than half of the participants, and had different screening intentions. These results suggest the importance of a differentiated approach in considering adoption stages.

Age was an important factor in separating individuals who made the decision to be screened from those who actually went ahead with the screening (in action). Those who were aged 20-30 years were more likely to be in stage 1 and 2 (for the adoption of mammography), while those who were aged 40-50 years were more likely to be in stage 6 and 7. This is consistent with previous studies (Lee et al., 2010; Samah and Ahmadian, 2012) showing that older women were more likely to exhibit breast health-related behaviors than younger women. This may be explained by the fact that women over 40 years old have access to preventive breast health care, through breast cancer screening during life in transition provided by the National Health Insurance Corporation (2009). Unmarried women were more likely to be in stages 1 and 2, while married women were more likely to be in stages 6 and 7. These results are consistent with previous studies, confirming that married women had more experience with early breast cancer screening than single women (Doganer et al., 2014), and that spousal support is an important facilitating factor in performing regular BSE (Dahlui et al., 2012). However, the incidence of breast cancer in individuals of age 30 and lower in Korea is steadily increasing. Based on our findings, young women are less likely to have the awareness and attention to engage in breast cancer screening behavior. We propose that young women be actively engaged in breast cancer screening, and be guided on screening behavior. Among the facilitating factors, exposure to mass media messages related to the hazards of and precautions against breast cancer was the most important factor in progressing to stage 2 from stage 1 (adoption of mammography). This was consistent with previous reports by Weinstein et al. (2008). In this study, those who had never heard about mammography accounted for over $11 \%$ of participants; thus, it seems necessary to take actions towards developing highly effective methods by using the mass media to raise, awareness on breast cancer screening, in order to change health behavior. Furthermore, among those who had been thinking about getting screened (stage 3 ), having a provider recommendation or the exposure to media messages about breast cancer was more likely to be reported compared to those who had never heard about screening (stage 1) or had not been thinking about getting screened (stage 2). Thus, our data suggest that recommendation by an acquaintance or a health professional, or mass media messages may be helpful in getting individuals to think about being screened.

With regard to the prediction of health beliefs, our study showed that perceived severity can predict progression of the stage of adoption of breast cancer screening and was an important predictor in progressing to stage 3 from stage 2 (for the adoption of BSE). Within those who had been thinking about performing BSE (stage 3 ), severity was more likely to be perceived compared to those who had not been thinking about performing BSE (stage 2). Additionally, the perceived severity of BSE was significantly higher in the undecided, action, and maintenance stages than in the unengaged stage. These results are similar to the findings of Erbil and Bolukbas (2014), who reported that a higher perceived severity leads to preventive breast cancer behavior. However, our findings are not consistent with those of Weinstein et al (2008), which demonstrated that beliefs such as susceptibility, severity, effectiveness, and difficulty were important predictive factors that determined progress between stage 3 and stage 4 or stage 5. Notably, however, in our study, the characteristics of stage 4 were not properly reflected, because there were not enough women in this group for meaningful analysis. We propose that a future study be conducted that includes sufficient numbers of participants for all the stages.

Self-efficacy was an important predictor in progressing from 5 to stage 6 (both BSE and mammography), and was significantly higher in the maintenance stage compared to other stages. This finding is consistent with other studies, which noted that a higher self-efficacy is observed with increasing stage of breast cancer screening adoption (Russell et al., 2007). Another study reported that selfefficacy of cancer screening is an important predictor in the progression from stage 5 to stages 6 and 7 (Kye et al., 2006). Breast cancer screening is not a one-time or intermittent activity, but rather is health behavior that must be consistently maintained. Park et al. (2009) reported that the degree of self-efficacy decreases with time. Sustaining 
self-efficacy is the key to continuous breast cancer screening practice. Thus, we suggest that people who are in the lower stages of practical action should be motivated to continue breast cancer screening. The strengthening of self-efficacy can be achieved through specific, accurate information transmission and continuous education to enable the correct understanding about the effectiveness of breast cancer screening.

In this study, PAPM stage analysis was limited because there were very few individuals who firmly decided not to be screened (stage 4). They are a particularly difficult group, who tend to dispute or ignore information that challenges their decision not to act, but they are also an important group to understand. Therefore, future studies should address the issues involved. Additionally, our findings were consistent with only some of the data of Weinstein et al. (2008). Nonetheless, our study may be useful for several reasons. First, this is the first study that applies PAPM to understanding breast cancer screening behaviors (for the adoption of BSE and mammography) for the Korean population in a community setting. Second, our study allows practitioners to respond while considering each individual's characteristics and stages of breast cancer screening adoption.

In conclusion, our results demonstrate that variables such as age, marital status, exposure to media about breast cancer, self-efficacy, and perceived severity are factors affecting the progress of PAPM stage of breast cancer screening (BSE and mammography). Our findings may be helpful for the development of intervention strategies to improve Korean women's breast cancer screening practices, such as using an appropriate media utility to spread a tailored message, or using self-efficacy enhancement programs for encouraging the sustained action of breast cancer screening.

\section{References}

Baysal HY, Polat H (2012). Determination of the breast cancer risk levels and health beliefs of women with and without previous mammography in the eastern part of Turkey. Asian Pac J Cancer Prev, 13, 5213-7.

Blalock SJ, DeVellis RF, Giorgino KB, et al (1996). Osteoporosis prevention in premenopausal women: using a stage model approach to examine the predictors of behavior. Health Psyc, 15, 84-93.

Champion VL (1984). Instrument development for health belief model constructs. Adv Nurs Sci, 6, 73-85.

Champion VL (1993). Instrument refinement for breast cancer screening behaviors. J Nurs Res, 42, 139-43.

Champion VL (1995). Development of a benefits and barriers scale for mammography utilization. Cancer Nurs, 18, 53-9.

Champion VL (1999). Revised susceptibility, benefits, and barriers scale for mammography screening. Res Nurs Health, 22, 341-8.

Champion VL, Skinner CS, Menon U (2005). Development of a self-efficacy scale for mammography. Res Nurs Health, 28, 329-36.

Costanza ME, Luckmann R, Stoddard AM, et al (2005). Applying a stage model of behavior change to colon cancer screening. Prev med, 41, 707-19.

Dahlui M, Gan DE, Taib NA, et al (2012). Predictors of breast cancer screening uptake: a pre intervention community survey in Malaysia. Asian Pac J Cancer Prev, 13, 3443-9.

Doganer YC, Aydogan U, Kilbas Z, et al (2014). Predictors affecting breast self-examination practice among Turkish women. Asian Pac J Cancer Prev, 15, 9021-5.

Erbil N, Bolukbas N (2014). Health beliefs and breast selfexamination among female university nursing students in Turkey. Asian Pac J Cancer Prev, 15, 6525-9.

Ergin AB, Sahin NH, Sahin FM, et al (2012). Meta analysis of studies about breast self examination between 2000-2009 in Turkey. Asian Pac J Cancer Prev, 13, 3389-97.

Han MY, Chung CW (2006). Breast cancer screening behaviors in working women. Korean J Women Health Nurs, 12, 363-70.

Jerome-D'Emilia B (2014). A systematic review of barriers and facilitators to mammography in hispanic Women.J Transcult Nurs, 26, 73-82.

Kim JK, Kwak BS, Lee JS, et al (2007). Do very young Korean breast cancer patients have worse outcomes? Ann Surg Oncol, 14, 3385-91.

Korean Breast Cancer Society (2013). Breast Cancer Facts \& Figures. Korea.

Kye SY (2009). A prediction model for stages of change in cancer screening. Unpublished doctor's dissertation. Ewha Women's University, Seoul.

Kye SY, Choi KS, Sung NY, et al (2006). Using the PAPM to examine factors associated with stages of adoption for stomach cancer screening. Korean J Health Educ Promot, 23, 29-45.

Lee CH, Kim HJ, Kim YI (2010). Factors affecting active early detection behaviors of breast cancer in outpatients. Korean $J$ Women Health Nurs, 16, 126-36.

Lee EH, Kim JS, Song MS (2002). Translation and validation of Champion's health belief model scale with Korean women. Cancer Nurs, 25, 391-5.

Ministry of Health and Welfare (2013). 2012 National cancer screening rate. Korea.

National Health Insurance Corporation (2009). Annual report on the diseases statistics. Korea.

Nattinger A (2010). In the clinic. Breast cancer screening and prevention. Ann Intern Med, 152, 41.

Park SM, Song HY, Hur HK, et al (2009). Effects of a cognition oriented breast self-examination intervention for korean women and their spouses. Public Health Nurs, 26, 259-68.

Russell KM, Monahan P, Wagle A, et al (2007). Differences in health and cultural beliefs by stage of mammography screening adoption in African American women. Cancer, 109, 386-95.

Samah AA, Ahmadian M (2012). Socio-demographic correlates of participation in mammography: a survey among women aged between 35- 69 in Tehran, Iran. Asian Pac J Cancer Prev, 13, 2717-20.

Suh Jh, Kim SB (2010). Early screening for breast cancer. Korean $J$ Med, 79, 237-40.

Weinstein ND, Lyon JE, Sandman PM, et al (1998). Experimental evidence for stages of health behavior change: the precaution adoption process model applied to home radon testing. Health Psychol, 17, 445-53.

Weinstein ND, Sandman PM, Blalock SJ (2008). The precaution adoption process model. In 'Health Behavior and Health Education: Theory, Research, and Practice', Eds Glanz K, Rimer BK and Viswanath K. Jossey-Bass Press, San Francisco, pp 123-47. 